\title{
Clinical factors associated with statins prescription in acute ischemic stroke patients: findings from the Lombardia Stroke Registry
}

Isabella Canavero ${ }^{*}$, Anna Cavallini ${ }^{1}$, Patrizia Perrone ${ }^{2}$, Mauro Magoni ${ }^{3}$, Lucia Sacchi ${ }^{4}$, Silvana Quaglini ${ }^{4}$, Giordano Lanzola ${ }^{4}$, Giuseppe Micieli ${ }^{1}$, on behalf of the Lombardia Stroke Registry (LSR) investigators

\begin{abstract}
Background: Statins, due to their well-established pleiotropic effects, have noteworthy benefits in stroke prevention. Despite this, a significant proportion of high-risk patients still do not receive the recommended therapeutic regimens, and many others discontinue treatment after being started on them. The causes of non-adherence to current guidelines are multifactorial, and depend on both physicians and patients. The aim of this study is to identify the factors influencing statin prescription at Stroke Unit (SU) discharge.

Methods: This study included 12,750 patients enrolled on the web-based Lombardia Stroke Registry (LRS) from July 2009 to April 2012 and discharged alive, with a diagnosis of ischemic stroke or transient ischemic attack (TIA) and without contra-indication to statin therapy. By logistic regression analysis and classification trees, we evaluated the impact of demographic data, risk factors, tPA treatment, in-hospital procedures and complications on statin prescription rate at discharge.

Results: We observed a slight increase in statins prescription during the study period (from 39.1 to 43.9\%). Lower age, lower stroke severity and prestroke disability, the presence of atherothrombotic/lacunar risk factors, a diagnosis of non-cardioembolic stroke, tPA treatment, the absence of in-hospital complications, with the sole exception of hypertensive fits and hyperglycemia, were the patient-related predictors of adherence to guidelines by physicians. Overall, dyslipidemia appears as the leading factor, while TOAST classification does not reach statistical significance.

Conclusions: In our region, Lombardia, adherence to guidelines in statin prescription at Stroke Unit discharge is very different from international goals. The presence of dyslipidemia remains the main factor influencing statin prescription, while the presence of well-defined atherosclerotic etiopathogenesis of stroke does not enhance statin prescription. Some uncertainties about the risk/benefit of statin therapy in stroke etiology subtypes (cardioembolism, other or undetermined causes) may partially justify the underuse of statin in ischemic stroke. The differences that exist between current international guidelines may prevent a more widespread use of statin and should be clarified in a consensus.
\end{abstract}

Keywords: Statins, Ischemic stroke, Adherence, Performance predictors, Classification tree

\footnotetext{
* Correspondence: isabellacanavero@libero.it

${ }^{1}$ Department of Emergency Neurology/Stroke Unit, National Neurologic Institute C. Mondino IRCCS, Pavia, Italy

Full list of author information is available at the end of the article
} 


\section{Background}

Statins have noteworthy benefits in stroke prevention as well as in cardiovascular diseases due to their wellestablished pleiotropic action [1]. In fact, beyond their well-known lipid-lowering effect, their role in the stabilization of endothelial function, in immunomodulation and antithrombotic processes, has been hypothesized and subsequently demonstrated [2-4]. After the SPARCL trial [5] many other studies have indicated that statins are of benefit to the majority of ischemic stroke patients as they reduce the risk of recurrence and improve outcome [6-13]. It has also been observed that daily intake of a statin after an acute ischemic stroke could improve functional outcome in patients with LDL levels $\leq 100 \mathrm{mg} / \mathrm{dL}$ [2]. The last Italian guidelines for stroke [14], in agreement with recommendations from other international guidelines $[15,16]$, state that statins are indicated in patients with ischemic stroke even in the absence of hypercholesterolemia. Moreover, the most recent ACC-AHA guidelines for management of high blood cholesterol levels [17], based on RCTs results, recommend a high-intensity statin therapy for every patient with "clinical atherosclerotic CVD" (ASCVD). ASCVD patients include acute coronary syndromes, a history of myocardial infarction, stable or unstable angina, coronary or other arterial revascularization, stroke, TIA or peripheral atherosclerotic arterial disease. In the presence of at least one of these clinical events, patients should receive statin therapy regardless LDL cholesterol levels [18].

Despite this scientific evidence and guideline recommendations, an unacceptably high proportion of stroke patients are neither on lipid-lowering therapy nor managed aggressively enough to achieve recommended target cholesterol levels $[19,20]$. Causes for non-adherence to current guidelines are multifactorial, and depend both on physicians and patients.

Understanding the gap between a physician's knowledge and his actual actions may be essential for the development of strategies aiming to improve patient management: predictors of adherence and causes for non-adherence should be identified and evaluated carefully.

Most studies have examined indirectly physicians' adherence to guidelines, through self-administered questionnaires and interviews. To our knowledge, there have been only a few attempts to describe the topic using data from real clinical practice (i.e., the prescribed therapies as they appear on the discharge letter), and to characterize the clinical factors interfering with statin prescription: the GWTG Stroke, the Swedish Stroke Register, the Paul Coverdell National Stroke Registry [21-23]. These registries describe prescription trends but are not able to identify the barriers to prescription.

The aim of this study is to identify the clinical factors influencing statin prescription by physicians in acute ischemic stroke patients at discharge from Lombardia Stroke Units [24]. We analyzed data collected from a webbased registry: the Lombardia Stroke Registry (LSR), describing the activity of our region's Stroke Units.

\section{Methods}

The study was based on data collected from July 2009 to April 2012 at 42 Stroke Units participating in the LSR. The LSR gathers demographic, clinical, and procedural data of acute stroke patients. Data-entry was performed by external staff, after training on how to retrieve data from hospital-specific clinical charts and documentation. In 40 of the 42 Stroke Units the treating specialty is neurology, in one internal medicine and in one the management switched from internal medicine to neurology during the study period. All the physicians operating in our Stroke Units are certified for the administration of the NIHSS and the modified Rankin scale (mRS).

For this study, we considered only the patients discharged alive, with a diagnosis of ischemic stroke or TIA, and without clinical contra-indications to statin prescription (e.g. hepatopathy, myopathy or hemorrhagic diathesis). They were divided into 2 groups: 'Statin +' group = patients discharged on statin therapy and 'Statin -' group = patients not discharged on statin therapy.

\section{Clinical variables}

The following variables were considered in the analysis: 1. demographics (age, gender); 2. prestroke and discharge disability (evaluated by $\mathrm{mRS}$ ); 3 . vascular risk factors and comorbidities (previous TIA/stroke, arterial hypertension, diabetes mellitus, dyslipidemia, atrial fibrillation, myocardial infarction, coronary artery disease, peripheral artery disease, smoking, heart failure, cognitive impairment, prosthetic cardiac valve); 4. stroke severity at admission and at discharge (evaluated by the National Institute of Health Stroke Scale - NIHSS score); 5. emergency treatment (intravenous or intra-arterial thrombolysis); 6. in-hospital neurological and medical complications (defined as the occurrence or absence of the following events: intracranial hypertension, hypertensive fits, seizures, bleeding, hypoxemia, hyperglycemia, falls, psychiatric disorders, deep venous thrombosis/pulmonary embolism, atrial flutter/fibrillation, acute myocardial infarction, bedsores, ventricular arrhythmias, fever, pneumonia, urinary infections); 7. stroke subtype (TIA or ischemic stroke) and etiopathogenetic classification according to TOAST criteria [25]; 8. statin and other secondary prevention therapies at discharge.

Risk factors/comorbidities were considered as present if the factor was documented in past medical history or the patient was on drug treatment for a specific comorbidity. 


\section{Statistical analysis}

Patients were coded as $1=$ Statin + and $0=$ Statin - . All the variables were coded as: $1=$ present, $0=$ absent. Age was codified as 0 if age $<80 \mathrm{yrs}$ and as 1 if age $>=80$ yrs; gender was codified as $0=$ female and $1=$ male. mRS score was discretized into two levels: no disability $(\mathrm{mRS}<2)$ and disability $(\mathrm{mRS}>=2)$. No disability was assigned the code 0 while disability was coded as 1 . NIHSS score was recoded as 0 for values $\leq 8$ and 1 for values $>8$. Differences between the two groups of patients, stratified for Statin + and Statin-, were analyzed by a Chi square test for categorical variables. To build a predictive model for statin prescription at discharge, a multivariate logistic regression was performed through a stepwise analysis. First, we performed two separate analyses for the prestroke and in-hospital variables, then we evaluated an overall model. All the analyses were performed with S-Plus [26]. The logistic regression model highlights the variables that are significant independent predictors of statin prescription. However, the variable combination leading to statin prescription is not explicitly identified, even if it could be useful in analyzing physicians' motivations for non-adherence to guidelines. Thus, in addition to logistic regression we exploited machine learning (ML) techniques to describe the possible care paths that lead to a specific outcome (prescription/non-prescription). In particular, we applied the ML 'Classification Tree' algorithm to the set of variables identified by the logistic regression analysis.

Classification Trees are algorithms that work by recursively partitioning the examples space, based on the most discriminant feature in order to predict the 'class variable' (i.e. statin prescription). Thanks to their structure, Classification Trees allow easy visualization, exploration and interpretation of results. In our study, each branch of the tree identifies a set of conditions (e.g. age, risk factors, treatments) that define subsets of patients where statin prescription is more or less likely.

For the construction of Classification Trees we used the algorithm that is implemented in the Orange Data Mining Suite $[27,28]$. This study was approved by the Ethics Committees of all of the institutes co-operating in the Lombardia Stroke Network (see Acknowledgements for details). Written informed consent for the handling of personal data was obtained from all subjects.

\section{Results}

Out of a total of 16,002 patients in the registry, 12,873 were discharged alive with a diagnosis of ischemic stroke or TIA. Out of these, 123 (0.95\%) had contra-indications to the prescription of statins and were not included in the analysis. Thus, 12,750 patients meeting the inclusion criteria were analyzed: 5392 (42\%) belong to the Statin + group and $7358(58 \%)$ to the Statin - group.
The percentage of patients discharged with statin therapy was $39.1 \%$ in $2009,41.9 \%$ in $2010,42.7 \%$ in 2011 and $43.9 \%$ in 2012, showing a slight upward trend.

\section{Prestroke profiles}

Table 1 was designed to study the predictive value of the individual patients' demographic and prestroke clinical features. The table shows the percentage of statin prescription given the values of the different predictors considered. For example, out of 6779 males, 3107 (46\%) underwent statin prescription. Out of $(5971=12750$ 6779) females, 2285 (38\%) underwent statin prescription. The percentage of prescription in males is significantly higher than that in females, as shown by the p-value in the last column.

In addition to male gender, patients with the following characteristics were more likely to be treated with statins: lower age, lower prestroke mRS score, personal history of hypertension, diabetes, myocardial infarction (MI), dyslipidemia, coronary artery disease (CAD), peripheral artery disease (PAD), smoking, and absence of personal history of atrial fibrillation (AF) or cognitive impairment. At multivariate analysis, considering these prestroke variables, the independent ones predicting statin prescription at discharge were (sorted by the variable influence on prescription):

- personal history of dyslipidemia (OR 3.71, 95\% CI 3.25-4.26, p value $<0.00001$ )

- personal history of myocardial infarction (OR 2.01, 95\% CI 1.69-2.39, p value $<0.00001$ )

- age (OR 0.54, 95\% CI 0.48-0.61, p value < 0.00001 )

- personal history of PAD (OR 1.74, 95\% CI 1.42-2.13, $\mathrm{p}$ value $<0.00001)$

- prestroke mRS (OR 0.68, 95\% CI 0.59-0.78, $\mathrm{p}$ value $<0.00001)$

- personal history of atrial fibrillation (OR 0.70, 95\% CI 0.61-0.81, p value $<0.0001)$

- personal history of CAD (OR 1.24, 95\% CI 1.01-1.53, p-value $=0.04$ )

- personal history of smoking (OR 1.19, 95\% CI $1.03-1.38$, $\mathrm{p}$ value $=0.01$ )

- personal history of diabetes (OR 1.15 CI 1.02 - 1.31, $\mathrm{p}$ value $=0.02$ )

- personal history of hypertension (OR 1.15, 95\% CI $1.04-1.28, \mathrm{p}$ value $=0.008)$

\section{In-hospital phase}

As shown in Table 2, at the emergency department 5.0\% $(\mathrm{n}=636)$ of patients received thrombolysis treatment (544 patients were treated with intravenous thrombolysis and 92 with intra-arterial thrombolysis). Statins were prescribed in 294 (54\%) patients who had been treated with intravenous thrombolysis, and in 37 (40\%) patients 
Table 1 Statin prescription according to demographics, prestroke disability and risk factors/comorbidities

\begin{tabular}{|c|c|c|c|c|}
\hline \multirow[b]{2}{*}{ Gender, n (\%) } & \multirow{2}{*}{$\begin{array}{c}\text { All (12750) } \\
\text { Male: } 6779(53 \%)\end{array}$} & \multicolumn{2}{|c|}{ Statin $+(5392,42 \%)$} & \multirow{2}{*}{$\frac{p \text {-value }\left(\mathbf{x}^{\mathbf{2}}\right)}{p<0.0001}$} \\
\hline & & Male & Female & \\
\hline & & 3107 (46\%) & 2285 (38\%) & \\
\hline \multirow[t]{2}{*}{ Age, n (\%) } & Age <80: 8889 (70\%) & Age $<80$ & Age $\geq 80$ & $p<0.0001$ \\
\hline & & 4251 (48\%) & $1141(30 \%)$ & \\
\hline \multirow[t]{2}{*}{ Prestroke mRS, $\mathrm{n}^{\S}(\%)$} & mRS 0-1: 5719 (80\%) & mRS 0-1 & $m R S 2-5$ & $p<0.0001$ \\
\hline & mRS 2-5: 1469 (20\%) & 2649 (46\%) & $468(32 \%)$ & \\
\hline Risk factors, n (\%) & & Rf present & Rf absent & \\
\hline Previous TIA/stroke & $2931(23 \%)$ & $1271(43 \%)$ & $4121(42 \%)$ & $p=0.18$ \\
\hline Arterial hypertension & 8166 (64\%) & $3628(44 \%)$ & $1764(38 \%)$ & $p<0.0001$ \\
\hline Diabetes mellitus & 2619 (21\%) & $1284(\mathbf{4 9 \% )}$ & 4108 (41\%) & $p<0.0001$ \\
\hline Myocardial infarction & $1407(11 \%)$ & 832 (59\%) & $4560(40 \%)$ & $p<0.0001$ \\
\hline Hypercholesterolemia & $2366(19 \%)$ & $1686(71 \%)$ & $3706(36 \%)$ & $p<0.0001$ \\
\hline Coronary artery disease & $999(8 \%)$ & 546 (55\%) & $4846(41 \%)$ & $p<0.0001$ \\
\hline Peripheral artheriopathy & $894(7 \%)$ & 462 (52\%) & $4930(42 \%)$ & $p<0.0001$ \\
\hline Atrial fibrillation & $2150(17 \%)$ & $720(34 \%)$ & $4672(44 \%)$ & $p<0.0001$ \\
\hline Smoking & $1804(14 \%)$ & 906 (51\%) & 4486 (41\%) & $p<0.0001$ \\
\hline Heart failure & $460(4 \%)$ & $190(41 \%)$ & $5202(42 \%)$ & $p=0.66$ \\
\hline Cognitive impairment & $967(8 \%)$ & $284(29 \%)$ & $5108(43 \%)$ & $p<0.0001$ \\
\hline Valvular prosthesis & $313(2 \%)$ & 137 (44\%) & $5255(42 \%)$ & $p=0.59$ \\
\hline
\end{tabular}

${ }^{\S}$ Not available for all patients.

treated with intra-arterial thrombolysis. Among patients who were not treated with thrombolysis, 5061 (42\%) received statin prescription at discharge. These percentages are significantly different $(\mathrm{p}<0.0001)$.

Patients with less severe neurological deficits, both at admission and at discharge, and minor disability at discharge, are more likely to receive statin prescription. The occurrence of complications during hospitalization (except for acute myocardial infarction, hypertensive fits and hyperglycemia) seems to lower the statin prescription rate. At discharge, the distribution of statin prescription was similar for ischemic stroke patients and TIAs. An etiopathogenetic diagnosis of cardioembolism made it less likely to have statin prescribed at discharge.

Considering the different TOAST etiopathogenesis, from Figure 1 we can see that statin prescription is low in all the diagnosis subtypes.

Comparing antithrombotic treatment at discharge, patients who did not receive any antithrombotics were less likely to be treated with statins.

At multivariate analysis, the in-hospital variables supporting statin prescription at discharge were (sorted by the variable influence on prescription):

- antithrombotic therapy at discharge (OR 2.65, 95\% CI 2.02-3.48, p-value $<0.00001$ )
- thrombolysis (OR 2.18, 95\% CI 1.69-2.81, $\mathrm{p}$ value $<0.00001)$

- pneumonia $(\mathrm{OR}=0.64,95 \%$ CI $0.47-0.89$, $\mathrm{p}$-value $=0.008)$

- hyperglycemia (OR 1.5, 95\% CI 1.22-1.84, $\mathrm{p}$ value $<0.0001)$

- intracranial hypertension $(\mathrm{OR}=1.42,95 \% \mathrm{CI}$ 1.04- 1.96 , p-value $=0.03$ )

- cardioembolism $(\mathrm{OR}=0.72,95 \%$ CI 0.64-0.82, p-value $<0.00001)$

- urinary infections $(\mathrm{OR}=0.75,95 \% \mathrm{CI}$ 0.62-0.92, $\mathrm{p}$-value $=0.006)$

- hypertensive fits (OR 1.29, 95\% CI 1.12-1.5, $\mathrm{p}$ value $=0.0006)$

- NIHSS at admission (OR 0.80, 95\% CI 0.67-0.96, $\mathrm{p}$ value $=0.016)$

- $\mathrm{mRS}$ at discharge $(\mathrm{OR}=0.87,95 \%$ CI 0.77-0.99, $\mathrm{p}$-value $=0.04$ )

\section{Predictors of statin prescription at discharge}

Considering all the variables without distinguishing between prestroke and in-hospital phases, we obtained the following overall stepwise logistic regression model (sorted by the variable influence on prescription):

- personal history of dyslipidemia (OR 3.88, 95\% CI 3.3 - 4.56, $\mathrm{p}$ value $<0.00001$ ) 
Table 2 Hospitalization: stroke severity, neurological and medical complications, disability at discharge

Emergency treatment:
Intravenous thrombolysis
Intra-arterial thrombolysis
No thrombolysis
Stroke severity (NIHSS):
At admission available in

At admission available in 8470 pts

At discharge available in 8132 pts

mRS at discharge available in 7958 pts

\section{In-hospital complications,} n (\%)

Intracranial hypertension

Acute myocardial infarction

Seizures

Hypoxemia

Hypertensive fits

Hyperglycemia

Atrial fibrillation/flutter

Fever

Ventricular arrhythmia

Bedsores

Deep venous thrombosis/ pulmonary embolism

Bleedings

Urinary infections

Falls

Pneumonia

Psychiatric disorders

Diagnosis at discharge

Ischemic stroke/TIA

TOAST classification available in 8564 patients

Cardioembolism
(possible/probable)
Non-cardioembolism:
-Large vessels
atherosclerosis
-Small vessels disease
-Other causes
-Undetermined etiology

All $(\mathbf{n}=\mathbf{1 2 7 5 0}$
$544(4.3 \%)$
$92(0.7 \%)$
$12114(95 \%)$

NIHSS $\leq 8: 6199$ (73\%)

NIHSS >8: 2271 (27\%)

NIHSS $\leq 8: 6691$ (82\%)

NIHSS >8: 1441 (18\%)

mRS 0-1: 3487 (44\%)

mRS 2-5: 4471 (56\%)

mRS 0-1

1707 (49\%)

Present

$170(40 \%)$

22 (59\%)

91 (34\%)

$250(32 \%)$

1060 (47\%)

579 (51\%)

324 (35\%)

$683(35 \%)$

$24(42 \%)$

$40(20.5 \%)$

16 (33\%)

182 (33\%)

431 (34\%)

$69(44 \%)$

$130(31 \%)$

$193(39.5 \%)$

\section{Ischemic \\ stroke}

$10534(83 \%)$
Statin +

294 (54\%)

37 (40\%)

$5061(42 \%)$

8

(

)

\begin{tabular}{|c|c|}
\hline 427 (3.5\%) & $170(40 \%)$ \\
\hline 37 (0.3\%) & 22 (59\%) \\
\hline $270(2 \%)$ & 91 (34\%) \\
\hline $782(6 \%)$ & $250(32 \%)$ \\
\hline 2240 (18\%) & 1060 (47\%) \\
\hline $1136(9 \%)$ & 579 (51\%) \\
\hline 931 (7\%) & $324(35 \%)$ \\
\hline 1922 (15\%) & $683(35 \%)$ \\
\hline $57(0.5 \%)$ & $24(42 \%)$ \\
\hline 195 (1.5\%) & $40(20.5 \%)$ \\
\hline $49(0.4 \%)$ & 16 (33\%) \\
\hline $547(4 \%)$ & 182 (33\%) \\
\hline 1276 (10\%) & 431 (34\%) \\
\hline $158(1 \%)$ & 69 (44\%) \\
\hline $419(3 \%)$ & $130(31 \%)$ \\
\hline \multirow[t]{2}{*}{488 (4\%) } & 193 (39.5\%) \\
\hline & $\begin{array}{l}\text { Ischemic } \\
\text { stroke }\end{array}$ \\
\hline $0534(8$ & $4482(43 \%)$ \\
\hline
\end{tabular}
3083 (36\%) With* other indications to prescription: 1059; without 2024
5481 (54\%) With* other indications to prescription: 1819; without 3662
2078 (24\%) With* other indications to prescription: 766; without 1312
1983 (23\%) With* other indications to prescription: 668; without 1315
178 (2\%) With* other indications to prescription: 43; without 135

1242 (15\%) With* other indications to prescription: 342; without 900
All

$4482(43 \%)$

$1113(36 \%)$

2607 (47\%)

1077 (52\%)

$979(49 \%)$

$48(27 \%)$

503 (40\%)

\section{NIHSS $>8$}

849 (37\%)

$p<0.0001$

544 (38\%)

$p<0.0001$

mRS 2-5

$p<0.0001$

1847 (41\%)

Absent

$5222(42 \%)$

$p=0.29$

5370 (42\%)

$p=0.03$

5301 (42\%)

$p=0.004$

$5142(43 \%)$

$p<0.0001$

4332 (41\%)

$p<0.0001$

4813 (41\%)

$p<0.0001$

5068 (43\%)

$p<0.0001$

4709 (43\%)

$p<0.0001$

5368 (42\%)

5352 (43\%)

$p=0.97$

$p<0.0001$

5376 (42\%)

$p=0.17$

5210 (43\%)

$p<0.0001$

4961 (43\%)

$p<0.0001$

5323 (42\%)

$p=0.72$

5262 (43\%)

$\mathrm{p}<0.0001$

5199 (42\%)

$p=0.21$

TIA

906 (41\%)

$p=0.14$

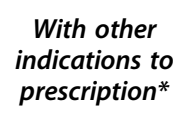

Without other
indications to prescription

542 (51\%)

571 (28\%)

$p<0.0001$

1185 (65\%)

1422(39\%)

504 (66\%)

573 (44\%)

$458(68 \%)$

$521(40 \%)$

$24(56 \%)$

$24(18 \%)$

199 (58\%)

304 (34\%) 
Table 2 Hospitalization: stroke severity, neurological and medical complications, disability at discharge (Continued)

\begin{tabular}{|c|c|c|c|}
\hline \multicolumn{4}{|c|}{$\begin{array}{l}\text { Antithrombotic treatment } \\
\text { at discharge }\end{array}$} \\
\hline Antiplatelet & 9763 (76\%) & 4296 (44\%) & $p<0.0001$ \\
\hline Anticoagulant & $2130(17 \%)$ & 933 (42.8\%) & \\
\hline None & 857 (7\%) & 163 (19\%) & \\
\hline
\end{tabular}

${ }^{*}=$ dyslipidemia, myocardial infarction, coronary artery disease (CAD), peripheral artery disease (PAD).

- antithrombotic therapy at discharge (OR 2.72, 95\% CI 2.03-3.66, p-value < 0.00001)

- personal history of myocardial infarction (OR 1.92, 95\% CI 1.56-2.37, $\mathrm{p}$ value $<0.00001)$

- age (OR 0.55, 95\% CI 0.48-0.64, p value $<0.00001$ )

- personal history of PAD (OR 1.64, 95\% CI 1.29-2.09, $\mathrm{p}$ value $<0.0001)$

- thrombolysis (OR 1.64, 95\% CI 1.24-2.17, $\mathrm{p}$ value $=0.0005)$

- pneumonia $(\mathrm{OR}=0.64,95 \%$ CI 0.44-0.91, $\mathrm{p}$-value $=0.015)$

- hypoxemia (OR 0.69, 95\% CI 0.52-0.92, $\mathrm{p}$ value $=0.01$ )

- personal history of atrial fibrillation (OR 0.73, 95\% CI 0.6-0.89, $\mathrm{p}$ value $=0.002)$

- prestroke mRS (OR 0.78, 95\% CI 0.67-0.92, $\mathrm{p}$ value $=0.003)$

- hypertensive fits (OR 1.28, 95\% CI 1.09-1.51, $\mathrm{p}$ value $=0.002)$

- NIHSS at discharge (OR 0.79, 95\% CI 0.66-0.95, $\mathrm{p}$ value $=0.013)$
- hyperglycemia (OR 1.22, 95\% CI 0.96-1.55, $\mathrm{p}$ value $=0.01$ )

- personal history of diabetes (OR 1.2 CI 1.03-1.41, $\mathrm{p}$ value $=0.02)$

- cardioembolism $(\mathrm{OR}=0.86,95 \%$ CI 0.73-1, p-value $<0.06)$

Figure 2 shows the Classification Tree obtained using the variables selected by the overall logistic regression analysis. This picture can be used in order to better explain the analysis results.

Dyslipidemia, at the top of the graph, is the most informative parameter in predicting statin prescription. If present, statins are prescribed in more than $70 \%$ of cases.

In the absence of dyslipidemia, the most informative indicator is the prescription of antithrombotic therapy: about $84 \%$ of the patients who are not undergoing antithrombotic treatment are not undergoing statin prescription either.

Also for patients under antithrombotic treatment, Statin- is the majority class. However, the tree shows

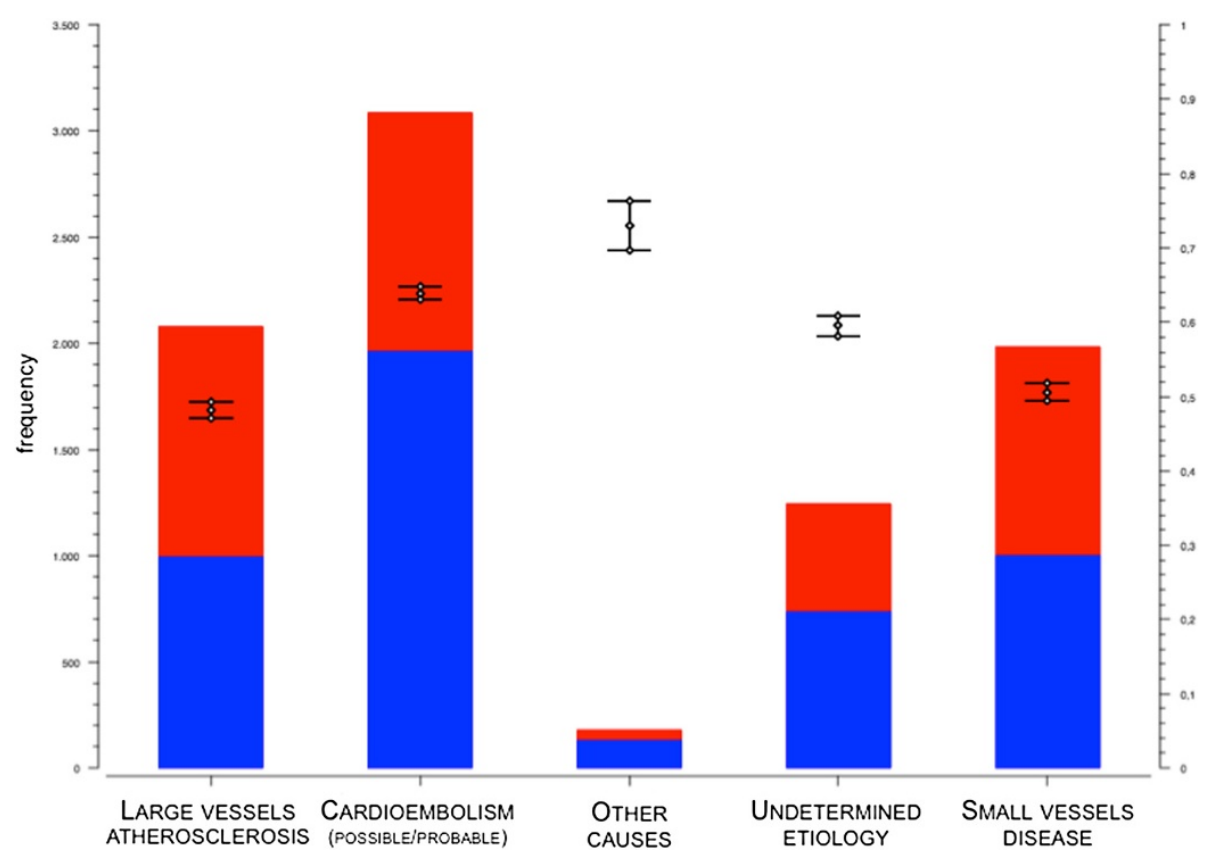

Figure 1 Statin prescription (red: presence, blue: absence) according to stroke etiology. 


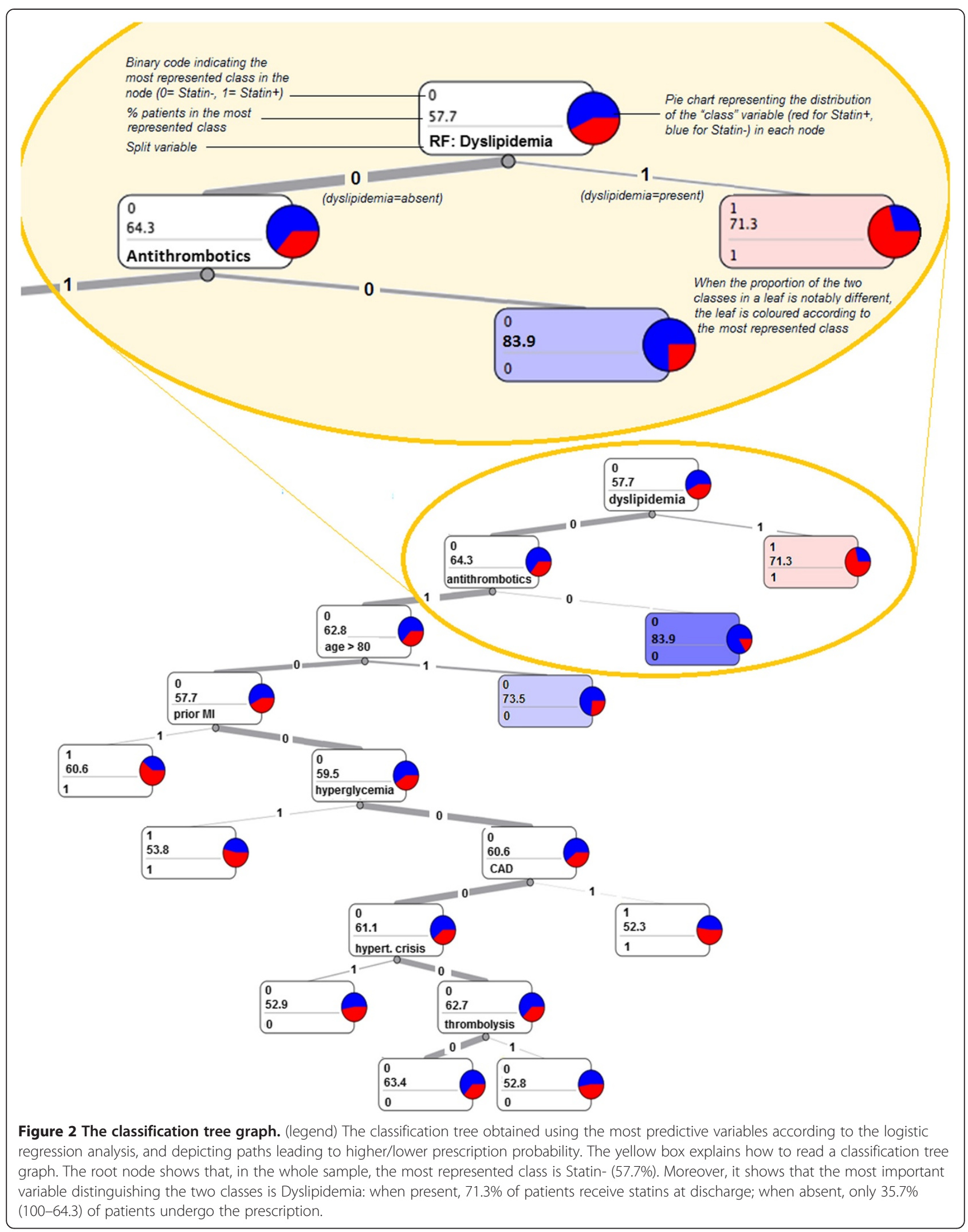


three groups of patients where Statin + is the majority class: patients aged less than 80 who had a prior $\mathrm{MI}$, those who experienced hyperglycemic episodes during the hospital stay and those affected by CAD.

\section{Subgroup analysis}

Considering the almost equal distribution of statin prescription across TOAST groups (Figure 1), we were not expecting TOAST classification to appear in the top portion of the classification tree. However, since the presence of Large Vessels Disease (LVD) is the main indication for statins and several works including the SPARCL trial [5] consider patients with stroke/TIA with non-cardioembolic stroke, we investigated this group of patients in a separate classification tree. As shown in Figure 3, the leading factor in statin prescription is the presence of dyslipidemia, as it is for the whole population.

Considering LVD patients without dyslipidemia (right branch of the tree), the algorithm identified age as the most informative parameter in the following decision process. In the majority of elderly patients, statins are not prescribed while for younger patients, the proportion of statin prescription and statin non-prescription is almost identical. Thus, from our data, we cannot identify any factors determining the choice of whether or not to prescribe statins in younger patients without dyslipidemia, even in the presence of well-defined atherosclerosis.

\section{Discussion}

In the literature there is a growing body of papers concerning the factors that can influence physicians' attitude to the prescription of new drugs. Statins are, in fact, quite old drugs, whose range of clinical indications has broadened after the recent RCTs.

After the publication of the SPARCL trial results in 2006, a progressive increase in the number of patients who receive statin therapy at discharge in the United States has been reported. In the GWTG-Stroke study, the percentage of statin prescription increased from $75.7 \%$ in 2005 to $83.5 \%$ in 2007 , however it was not considered high enough [21]. In our study, the percentage of patients treated with statins is only $42 \%$, with a slight increase during the study period. Few other settings show worse results than ours. For example, in a recent meta-analysis conducted in China, only $24 \%$ of ischemic stroke patients were treated with statins [29]. It has been hypothesized that epidemiological features of Chinese people - lower mean blood cholesterol levels, more frequent hemorrhagic complications than Western people could play a role in physicians' decision-making process.

Prescription decisions are affected by many factors related to physicians' attitudes and patient characteristics. Concerning physician-related factors, some authors have identified as good predictors of adherence to guidelines the habitual reading of medical journals and maintenance of professional contacts, such as talking to colleagues and attending scientific meetings [30]. It is also widely recognized that new drugs and guidelines are adopted earlier by specialists [30] and that clinical trial investigators are usually more prone to follow guidelines because of their link to the world of research [31]. Another study describes the so-called 'therapeutic traditions' which seem to influence the prescription behavior of individual physicians [32]. All these factors are difficult to quantify. As for patientrelated factors, those able to influence their attitude to comply with therapy (after discharge) are usually identified in the literature as patients' demographic, social and cognitive features. On the contrary, clinical factors detected during hospitalization are not usually considered as being among those that influence physicians' prescription.

To overcome this limitation, our work tried to identify those patient-specific clinical and care factors that are able to influence physicians' decisions to prescribe statin after an acute ischemic stroke. In our study, patients were more likely to be treated with statins if they were younger than 80 years old and not disabled before

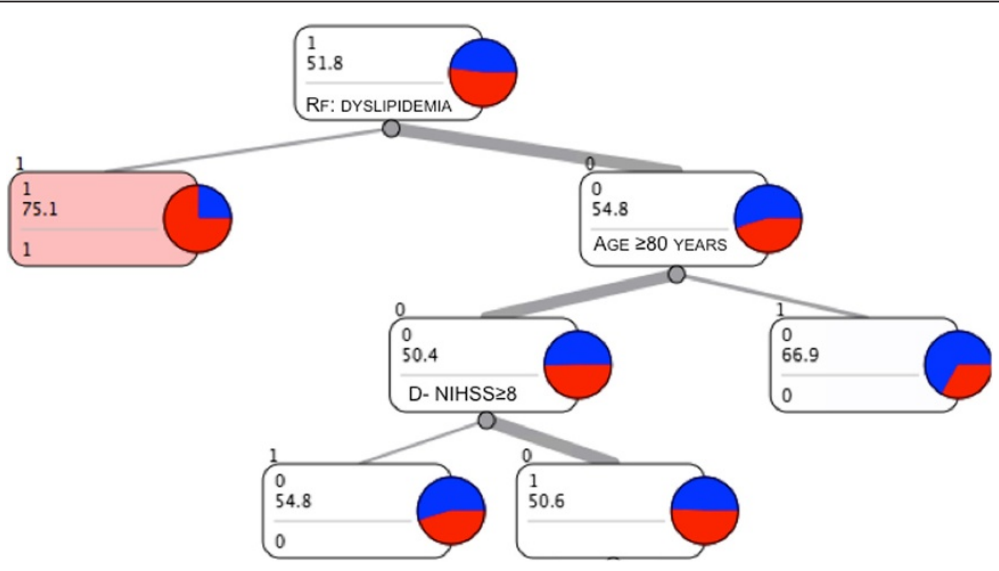

Figure 3 Classification tree graph for the subgroup Large Vessels Disease (LVD). 
stroke, they had a personal history of risk/disease of systemic atherosclerosis (dyslipidemia, diabetes, myocardial infarction, coronaropathy, peripheral arteropathy), they were treated with thrombolysis at the emergency department and discharged on antithrombotics for the secondary prevention of ischemic stroke.

It is interesting to note that, in our study, dyslipidemia represents the principal factor leading to the prescription of statin in ischemic stroke of all subtypes, including in LVD stroke.

Among demographics, older age, previous disability and lower social status, have been previously reported to negatively influence the adherence to guidelines in many settings [33]. According to the Swedish Stroke Register [22] the prescription of statins was strongly age-related: statin therapy, even if associated with reduced risk of death, seemed to be underused among elderly patients, with an inverse correlation with increasing age. In line with these findings, in our setting younger patients and those without prestroke disability were more likely to be treated with statins. A higher risk of hemorrhagic complications in older people could explain this trend, even if other authors have not found a different rate of side effects compared to younger patients [34]. It has been suggested that older people [35] and people with prior disability [36] tend to discontinue treatment during follow up. The hypothetical reduced compliance could, at least partially, explain the low frequency of prescription in these subjects. Another important point is that most of the current evidence derives from RCTs based on patients with an average age of 65 years or younger and little comorbidity [5]. Thus, the observed lower prescription rate in older and disabled patients may perhaps be a consequence of this signaling. Moreover, current literature on statins efficacy shows controversial results: a recent work examined the impact of statins on outcome in older ischemic stroke patients, finding benefits in functional outcome and 12-month survival [34]; another paper analyzed the effect of statins in the elderly in the prevention of $\mathrm{CV}$ events, finding a lower rate of MI and stroke but worse survival rate [37]. Regarding gender, it has been already reported that female patients are often undertreated [33,38]; our study confirms this trend. Probably, the well-known higher risk of cardio-cerebrovascular pathologies in men than in women plays a role in the prescription-making process, even if it cannot completely justify it.

Stroke severity, directly linked to disability and life expectancy, is another factor our model found as influencing prescription. Patients with lower NIHSS scores both on admission and at discharge are more likely to receive statins. These findings suggest that physicians tend to prescribe statins in patients with milder neurological damage, perhaps in order to obtain better compliance. Higher severity could thus represent a 'non-prescription' bias leading physicians to avoid secondary preventive therapies in patients who have had severe strokes and with poor prognosis. Then, as already pointed out, patients with significant disability and poor prognosis are excluded from the dedicated RCTs, whose results cannot be applied to them. Another possible explanation could be linked to the highest risk of hemorrhagic transformation characterizing patients with more severe neurological deficits [39]. A post hoc analysis of the SPARCL trial results found that the use of statins in stroke prevention was associated with an increase in the number of patients having hemorrhagic stroke [40]. Therefore, another factor in patients with higher stroke severity being less frequently treated with statins is the possibility of increased risk of hemorrhagic transformation in patients who are already susceptible.

We also found that patients who did not receive antithrombotics at discharge showed a low statin prescription rate. This could happen in a context of global non-adherence to guidelines. Alternatively, they could be untreated with antithrombotics because of a higher clinical severity (large infarction size, hemorrhagic transformation, etc.): probably physicians tend to avoid statin prescription in order to limit the possible higher risk of bleeding in the subgroup.

The presence of non-cardioembolic risk factors was among the main drivers of increased statin use in the GWTG-Stroke trial [21]: our results were in line with it. It is interesting to note that patients with hypertension and diabetes are more likely to be treated with statins than patients who are not affected by these risk factors, even if more than a half of them do not usually receive the prescription (see Table 1).

In the GWTG setting, patients with known dyslipidemia or who were on a cholesterol reducer at the time of hospital admission seem to be more likely to leave the hospital on a statin [21]. In our study too, comparing ORs, dyslipidemia shows the strongest correlation with statin prescription, followed by myocardial infarction and PAD; the Classification Tree confirmed dyslipidemia as the leading factor in prescriptive decisions. This means that dyslipidemia and cardiovascular pathologies still appear crucial in determining the need for statin therapy. Does this mean that stroke is not per se recognized as a vascular disease?

However, the relationship between total cholesterol levels and stroke outcome has not, as yet, been well characterized. Despite much clinical data supporting a linear relation between cholesterol lowering and cardiovascular risk reduction [41], in some papers hypercholesterolemia has been associated with better stroke outcome [42]. Recent works have described a ' $U$-shaped' association between cholesterol levels and stroke mortality, which was 
higher for patients with $<160 \mathrm{mg} / \mathrm{dl}$ [43]; the same cut-off was identified as a risk factor for a hemorrhagic fatal stroke [44].

Current guidelines for stroke [14-16] recommend statins for all patients with non-cardioembolic stroke. The best evidence from the literature derives from the SPARCL trial subanalysis: statins are mostly effective in reducing the risk of vascular events in patients with carotid atherosclerosis [45]. The last ACC-AHA guidelines [17] for lipid management identify "patients with clinical atherosclerotic cardiovascular disease" (including TIA and stroke) as the first subgroup of patients that should receive a high-intensity statin therapy, if tolerated.

In our setting, statin prescription rates for cardioembolism and 'other causes' of stroke are in line with current guidelines. However, it is particularly surprising that patients with large vessel disease, in the absence of other clinical indications for statins, have a prescription rate of only $44 \%$, despite the current guidelines strongly recommending statin therapy for atherosclerotic disease. The Classification Tree applied to the subgroup of LVD patients leads us to conclude that dyslipidemia is the leading factor for statin prescription, even in the presence of well-defined atherosclerosis, instead of recognizing LVD per se as the clinical indication for statin reception.

Concerning small vessel disease, the low rate of prescription we detected could be explained by the complex relationship between statins and lacunar stroke, and by the fact that literature lacks precise indications. Small vessel disease determines both ischemic (lacunar) and hemorrhagic (microbleeds) stroke, and severe arterial hypertension is the main risk factor in both cases. According to these factors, statins could somehow enhance the risk of hemorrhage in patients with lacunar stroke because of the underlying small vessel pathology and unbalanced hypertension. Recent papers have analyzed the connections between statins use and the risk of developing microbleeds, with non-univocal results. Day et al. [46] found that previous statin therapy was not associated with the prevalence or degree of microhemorrhages in patients with acute ischemic stroke or transient ischemic attack. Haussen et al. [47] found instead that statins use was independently associated with microbleeds in patients with ICH. Some authors [48] underline that patients with nonatherosclerotic causes of stroke should not be treated with statins and that statin therapy should be initiated only after a careful consideration of all the potential risks and benefits that could derive from them.

Besides the low rate of prescription in atherosclerotic disease, the Classification Tree does not highlight the TOAST criteria among the predictors of statin prescription: the therapy is prescribed mostly according to the risk profile.
Unexpectedly, thrombolytic treatment in the acute phase of stroke seems to enhance statin prescription. Results of studies about the association between statin therapy and thrombolysis are somehow controversial. Some studies analyze the effect of statin treatment before stroke and functional outcome after thrombolysis, with non-coherent findings: some have found a positive influence from statins [49], while others do not [50-52]. Engelter et al. [52] found that prior statins use was not an independent predictor of functional outcome or of $\mathrm{ICH}$ after thrombolysis, but it may be considered as an indicator of baseline characteristics that are associated with a less favorable course. Cappellari et al. [53,54] suggested that statin treatment initiated within 24 hours of IV thrombolysis, but not statin treatment started before stroke and continued in the acute phase, may positively influence functional outcome. It is important to note that most of papers analyze the relationship between prior statin therapy and the clinical outcome after thrombolysis while our work describes the rate of statin prescription after thrombolysis. Our results could be explained with Cappellari's findings. Further, to our knowledge, there is no evidence proving an increased risk of hemorrhage in patients treated with both rtPA and statins. We could also hypothesize that patients treated with thrombolysis are more likely to be managed following guidelines, and this could perhaps explain why they are more likely to receive the recommended statin regimen at discharge.

In the literature, statin therapy is mostly analyzed when administered during in-hospital stays. In this setting, it seems to be associated with a lower rate of complications in stroke patients. The prophylactic administration of statins significantly reduces in-hospital mortality [49] and is associated with a favorable outcome [55,56], with few incidences of adverse reaction [57]. Montaner et al. [58] reported that statin use between 3 and 12 hours after stroke was associated with neurological improvement at 3 days from ischemia, suggesting a rapid mechanism of action on coagulation or the fibrinolysis system. These findings should enhance physicians' attitude to statin prescription. Considering our available data (i.e., statin prescription rate at discharge), however, we cannot hypothesize that statins could determine a lower occurrence of complications, but that patients with inhospital complications are unlikely to be treated with statins, the only exception being hypertensive fits and hyperglycemia. This finding could be explained as previously done for age, disability and stroke severity: physicians avoid prescribing statins in critical patients, aside from situations that require statins for other indications than stroke. Besides, even if not confirmed by the logistic regression model, in our setting statins are not usually prescribed in patients with in-hospital 
bleeding, probably due to the higher risk of hemorrhage associated with statin use in the literature [59].

Besides these reports from the literature, we have to note that the effects of statins in acute stroke have been extrapolated - often with uncertain results - especially from retrospective studies, while few randomized clinical trials are currently available [60]. Consequently, current guidelines lack of some precise information, in particular concerning: their indication in non-atherothrombotic stroke [48], their recommended dose [60], the time to start therapy [8,58-62], their rate of side effects [12,40,57], the absolute contra-indications [48], the other concomitant therapies [12,49-53], the recommended approach with respect to old age (in RCTs concerning secondary prevention with statins the mean age is $<65$ years [12]).

In this paper we have not explored the physician-related factors influencing statin prescription but, reviewing the literature in order to compare our results with other experiences, we found more than a few grey areas among recommendations. We can hypothesize that the low statin prescription rate by physicians may be partially due to these controversies.

\section{Limits of the study}

This study has some limitations. First, patient factors such as patient preferences and agreement to treatment are not addressed. As a matter of fact, these variables are not usually collected in a registry, and thus this information was not available in this study. Also, this study only provides the categorical data of vascular risk factors (e.g. diabetes mellitus, dyslipidemia, etc.) rather than the raw values of the biological parameters (e.g. blood glucose, total cholesterol, HDL, LDL etc.). The LSR Registry is a repository where mostly "qualitative" and process information is stored, focusing on the presence of a diagnosis rather than on its entity. Then, type and dosage of statin is not precisely reported in LSR, which may cause potential bias in statistical analysis. This is due to the Registry structure, modeled on the form of current clinical practice guidelines for stroke that in fact are not specific as well, recommending to use a statin without specifying which type.

Moreover, we performed the analyses without considering the type of providing treatment specialist, which might impact on the prescription decision-making. The reason why we did not consider such parameter is that the distribution of treatment specialty is very uneven, with only one center managed by internal medicine specialists, one whose management shifted during the study period and 40 managed by neurologists. In the internal medicine Stroke Unit, the percentage of statin prescriptions was higher (65.7\%) than the overall average percentage (42\%), in the one where the management shifted from Internal Medicine to Neurology it was slightly above the average
(43.14\%). As regards the centers managed by neurologists, the statins prescription rate has a high variability, with values ranging from $15.8 \%$ to $82.8 \%$. From this data it is difficult to evaluate the role of the treating specialty as a predictor of prescription.

Although our Registry is provided with a follow-up section, in this study we have not included information on post-discharge statins use. The motivation underlying this choice is twofold. On the one hand, the follow-up is only partially completed for our patients. On the other hand, for those patients who died during follow-up, the registry doesn't allow physicians to enter information about treatments in use before death. This would cause a bias, as the patients who died would result as not continuing the treatment even if they were.

\section{Conclusions}

Our setting confirmed that adherence to guidelines regarding statin prescription rates by physicians is very different from international goals. Analyzing the decision-making process, we found that the presence of dyslipidemia - or at least a cardiovascular comorbidity appears as the main factor influencing statin prescription, even if current guidelines recommend the drug in any case of non-cardioembolic stroke. On the contrary, the presence of well-defined atherosclerosis does not enhance statin prescription. Some uncertainties about the risk/benefit of statin therapy and grey areas in certain subgroups of stroke patients may partially justify the low rate of prescription we detected.

These findings must be considered in devising approaches to enhance adherence to guidelines by physicians to improve the management of patients and compliance to the recommended therapies. We highlight the need for a consensus on the above-mentioned grey areas in order to achieve improved and detailed guidelines that are easier for physicians to put into practice.

\section{Competing interests}

No financial or non-financial competing interests to declare.

\section{Authors' contributions}

IC participated in the design of the study, in the statistical analysis, in review of the literature and drafted the manuscript. AC participated in the design of the original database, in the design of the study, in the statistical analysis, in the review of the literature and revised the manuscript. PP participated in the acquisition and interpretation of data. MM participated in the acquisition and interpretation of data. LS participated in the acquisition and interpretation of data and performed the statistical analysis (especially for Machine Learning techniques) and contributed to the manuscript drafting and revision. SQ participated in the design and realization of the original database, in the acquisition and interpretation of data and performed the statistical analysis and contributed to the manuscript drafting and revision. $G L$ participated in the design and realization of the original database, in the acquisition of data and in coordinating the LSR centers. GM participated in the design of the original database, conceived the study, participated in coordinating the LSR centers, and revised the manuscript. All authors read and approved the final manuscript. 


\section{Acknowledgements}

We would like to thank all the Lombardia Stroke Registry collaborators for their support and help in the realization of the LSR database. In alphabetical order:

Laura Adobbati ('Auxologico Italiano' IRCCS, Milan), Elio Agostoni ('Manzoni' Hospital, Lecco), Marco Arnaboldi ('Sant'Anna' Hospital, Como), Gianstefano Baietti (Cremona Hospital), Ubaldo Balducci (Chiari Hospital), Pierluigi Baron ('Ca' Granda' Policlinico, Milan), Pietro Bassi ('San Giuseppe' Hospital, Milan), Paola Bazzi (Vimercate Hospital), Simone Beretta (Monza Hospital), Luciano Bet ('San Donato' Hospital, Milan), Luigi Bettoni (Cremona Hospital), Giacomo Bezzi (Sondrio Hospital), Giorgio Boncoraglio ('Carlo Besta' IRCCS, Milan), Giorgio Bono ('Fondazione Macchi' Hospital, Varese), Bianca Bordo (Desio Hospital), Giuseppina Borutti (Voghera Hospital), Nereo Bresolin ('Ca' Granda' Policlinico, Milan), Fabio Brusaferri (Crema Hospital), Maria Vittoria Calloni (Legnano Hospital), Massimo Camerlingo ('San Marco' Policlinico, Zingonia), Bruno Censori ('Riuniti' di Bergamo Hospital), Carlo Cerini (Esine Hospital), Nicoletta Checcarelli ('Valduce' Hospital, Como), Francesco Chia (Chiari Hospital), Antonio Colombo (Desio Hospital), Giancarlo Comi ('San Raffaele' Hospital, Milan), Angelo Costa ('Spedali Civili' Hospital, Brescia), Vittorio Crespi (Vimercate Hospital), Maria Luisa Delodovici ('Fondazione Macchi' Hospital, Varese), Edoardo Donati ('Poliambulanza' Hospital, Brescia), Claudio Ferrante (Ponte San Pietro Hospital), Carlo Ferrarese (Monza Hospital), Fabio Frediani ('San Carlo Borromeo' Hospital, Milan), Paola Gambaro ('Luigi Sacco' Hospital, Milan), Antonella Gomitoni (Saronno Hospital), Giampiero Grampa (Saronno Hospital), Mario Guidotti ('Valduce' Hospital, Como), Patrizia Lattuada ('San Carlo Borromeo' Hospital, Milan), Ezio Lanza (Treviglio-Caravaggio Hospital), Andrea Magnoni (Merate Hospital), Simona Marcheselli ('Humanitas' Clinical Institute, Rozzano), Claudio Mariani ('Luigi Sacco' Hospital, Milan), Claudia Marsile (Vizzolo Predabissi Hospital), Alessandra Martignoni ('San Matteo' Policlinico IRCCS, Pavia), Marco Mattioli (Garbagnate Hospital), Giovanni Meola ('San Donato' Hospital, Milan), Graziella Molini (Vizzolo Predabissi Hospital), Irene Neromante (Busto Arsizio Hospital), Emanuele Poloni ('San Carlo Borromeo' Hospital, Milan), Eugenio Parati ('Carlo Besta' IRCCS, Milan), Giovanni Piccolo (Voghera Hospital), Marco Poloni ('Riuniti' Hospital, Bergamo), Daniele Porazzi (Busto Arsizio Hospital), Alessandro Prelle (Crema Hospital), Paolo Previdi (Mantova Hospital), Maurizio Riva (Lodi Hospital), Alessandro Romorini (Magenta Hospital), Ignazio Santilli ('Niguarda Ca' Granda' Hospital, Milan), Francesco Sasanelli (Vizzolo Predabissi Hospital), Chiara Scaccabarozzi ('Manzoni' Hospital, Lecco), Maria Sessa ('San Raffaele' Hospital, Milan), Vincenzo Silani ('Auxologico Italiano' IRCCS, Milan), Giorgio Silvestrelli (Mantova Hospital), Carlo Sebastiano Tadeo ('Città Studi' Clinical Institute, Milan), Marinella Turla (Esine Hospital), Simone Vidale ('Sant'Anna' Hospital, Como), Maria Teresa Zaccone ('Beato Matteo' Clinical Institute, Vigevano), Davide Zarcone (Gallarate Hospital), Angelo Zilioli (Lodi Hospital).

\section{Author details}

1Department of Emergency Neurology/Stroke Unit, National Neurologic Institute C. Mondino IRCCS, Pavia, Italy. ${ }^{2}$ Neurology Unit, Legnano Hospital, Legnano, Italy. ${ }^{3}$ Neurovascular Unit, 'Spedali Civili' Hospital, Brescia, Italy. ${ }^{4}$ Department of Electrical, Computer and Biomedical Engineering, University of Pavia, Pavia, Italy.

Received: 29 June 2013 Accepted: 17 March 2014 Published: 21 March 2014

\section{References}

1. Calabrò P, Yeh ET: The pleiotropic effects of statins. Curr Opin Cardiol 2005, 20(6):541-6.

2. Stead LG, Vaidyanathan L, Kumar G, Bellolio MF, Brown RD, Suravaram S, Enduri S, Gilmore RM, Decker WW: Statins in ischemic stroke: just low-density lipoprotein lowering or more? I Stroke Cerebrovasc 2009, 18(2):124-127.

3. Prinz V, Endres M: Statins and stroke: prevention and beyond. Curr Opin Neurol 2011, 24(1):75-80.

4. Cimino M, Gelosa P, Gianella A, Nobili E, Tremoli E, Sironi L: Statins: multiple mechanisms of action in the ischemic brain. Neuroscientist 2007, 13(3):208-13.

5. Amarenco P, Bogousslavsky J, Callahan A 3rd, Goldstein LB, Hennerici M, Rudolph AE, Sillesen H, Simunovic L, Szarek M, Welch KM, Zivin JA, Stroke Prevention by Aggressive Reduction in Cholesterol Levels (SPARCL)
Investigators: High-dose atorvastatin after stroke or transient ischemic attack. N Engl J Med 2006, 355(6):549-59.

6. Vergouwen MDI, De Haan RJ, Vermeulen M, Roos YB: Statin treatment and the occurrence of hemorrhagic stroke in patients with a history of cerebrovascular disease. Stroke 2008, 39(2):497-502.

7. Sacco S, Toni D, Bignamini AA, Zaninelli A, Gensini GF, Carolei A, the SIRIO Study Group: Effect of prior medical treatments on ischemic stroke severity and outcome. Funct Neurol 2011, 26(3):133-9.

8. Flint AC, Kamel H, Navi BB, Rao VA, Faigeles BS, Conell C, Klingman JG, Sidney S, Hills NK, Sorel M, Cullen SP, Johnston SC: Statin use during ischemic stroke hospitalization is strongly associated with improved poststroke survival. Stroke 2012, 43(1):147-54.

9. Biffi A, Devan WJ, Anderson CD, Cortellini L, Furie KL, Rosand J, Rost NS: Statin treatment and functional outcome after ischemic stroke case-control and meta-analysis. Stroke 2011, 42(5):1314-9.

10. Goldstein LB, Amarenco P, Zivin J, Messig M, Altafullah I, Callahan A, Hennerici M, MacLeod MJ, Sillesen H, Zweifler R, Michael K, Welch A: Statin Treatment and Stroke Outcome in the Stroke Prevention by Aggressive Reduction in Cholesterol Levels (SPARCL) Trial. Stroke 2009, 40(11):3526-31.

11. Milionis HJ, Giannopoulos S, Kosmidou M, Panoulas V, Manios E, Kyritsis AP, Elisaf MS, Vemmos K: Statin therapy after first stroke reduces 10-year stroke recurrence and improves survival. Neurology 2009, 72(21):1816-22.

12. Amarenco P, Labreuche J: Lipid management in the prevention of stroke: review and updated meta analysis of statins for stroke prevention. Lancet Neurol 2009, 8(5):453-63.

13. O'Regan C, Wu P, Arora P, Perri D, Mills EJ: Statin therapy in stroke prevention: a meta-analysis involving 121000 patients. Am J Med 2008, 121(1):24-33.

14. SPREAD (Stroke Prevention and Educational Awareness Diffusion) Guidelines. In 7th edition. Via Alberto Falck, 15, 20099 Sesto San Giovanni Milano: Pierrel Research Italy SpA. Version of $14^{\text {th }}$ March 2012. http://www. siapav.it/pdf/SPREAD\%202012.pdf.

15. Furie KL, Kasner SE, Adams RJ, Albers GW, Bush RL, Fagan SC, Halperin JL, Johnston SC, Katzan I, Kernan WN, Mitchell PH, Ovbiagele B, Palesch Y, Sacco RL, Schwamm LH, Wassertheil-Smoller S, Turan TN, Wentworth D, on behalf of the American Heart Association Stroke Council, Council on Cardiovascular Nursing, Council on Clinical Cardiology, and Interdisciplinary Council on Quality of Care and Outcomes Research: AHA/ASA Guideline Guidelines for the Prevention of Stroke in Patients With Stroke or Transient Ischemic Attack. A Guideline for Healthcare Professionals From the American Heart Association/American Stroke Association. Stroke 2011, 42(1):227-76

16. European Stroke Organisation (ESO) Executive Committee, ESO Writing Committee: Guidelines for management of ischaemic stroke and transient ischaemic attack 2008. Cerebrovasc Dis 2008, 25(5):457-507.

17. Stone NJ, Robinson J, Lichtenstein AH, Merz CN, Blum CB, Eckel RH, Goldberg AC, Gordon D, Levy D, Lloyd-Jones DM, McBride P, Schwartz JS, Shero ST, Smith SC Jr, Watson K, Wilson PW: 2013 ACC/AHA Guideline on the Treatment of Blood Cholesterol to Reduce Atherosclerotic Cardiovascular Risk in Adults: A Report of the American College of Cardiology/American Heart Association Task Force on Practice Guidelines. Circulation 2013 [Epub ahead of print].

18. Keaney JF Jr, Curfman GD, Jarcho JA: A pragmatic view of the new cholesterol treatment guidelines. N Engl J Med 2014, 370(3):275-8.

19. Castilla-Guerra L, Espino-Montoro A, Fernández-Moreno MC, López-Chozas JM, Jimernez MD: Statins and stroke. Current clinical practice. Stroke 2006, 37(5):1153.

20. Ovbiagele B, Saver JL, Bang H, Chambless LE, Nassief A, Minuk J, Toole JF, Crouse JR: Statin treatment and adherence to national cholesterol guidelines after ischemic stroke. Neurology 2006, 66(8):1164-70.

21. Ovbiagele B, Schwamm LH, Smith EE, Hernandez AF, Olson DWM, Pan W, Fonarow GC, Saver JL: Recent nationwide trends in discharge statin treatment of hospitalized patients with stroke. Stroke 2010, 41(7):1508-13.

22. Asberg S, Henriksson KM, Farahmand B, Asplund K, Norrving B, Appelros P, Stegmayr B, Asberg KH, Terént A: Ischemic stroke and secondary prevention in clinical practice: a cohort study of 14,529 patients in the Swedish stroke register. Stroke 2010, 41(7):1338-42.

23. Mullard AJ, Reeves MJ, Jacobs BS, Kothari RU, Birbeck GL, Maddox K, Stoeckle-Roberts S, Wehner S, Paul Coverdell National Acute Stroke Registry 
Michigan Prototype Investigators: Lipid testing and lipid-lowering therapy in hospitalized ischemic stroke and transient ischemic attack patients: results from a statewide stroke registry. Stroke 2006, 37(1):44-9.

24. Micieli G, Cavallini A, Quaglini S, Fontana G, Duè M: The Lombardia Stroke Unit Registry: 1-year experience of a web-based hospital stroke registry. Neurol Sci 2010, 31(5):555-64

25. Adams HP Jr, Bendixen BH, Kappelle LJ, Biller J, Love BB, Gordon DL, Marsh EE 3rd: Classification of subtype of acute ischemic stroke. Definitions for use in a multicentre clinical trial. TOAST. Trial of Org 10172 in Acute Stroke Treatment. Stroke 1993, 24(1):35-41.

26. Venables WN, Ripley BD: Modern applied statistics with S. Fourth Edition: Springer; 2002.

27. Demsar J, Zupan B: Orange: from experimental machine learning to interactive data mining. In White Paper (www.ailab.si/orange). Faculty of Computer and Information Science, University of Ljubljana; 2004.

28. Hastie T, Tibshirani R, Friedman J: The Elements of Statistical Learning: Data Mining, Inference, and Prediction. Secondth edition. Springer; 2009.

29. Li X, Gao Y, Li J, Feng F, Liu JM, Zhang HB, Zhang D, Hundei WH, Chen F, Chen YP, Chen ZM, Landray M, Armitage J, Jiang LX, Li LM, HPS2-THRIVE China group: Underuse of statins in patients with atherosclerotic ischemic stroke in China. Chin Med J (Engl) 2012, 125(10):1703-7.

30. Garjon FJ, Azparren A, Vergara I, Azaola B, Loayssa JR: Adoption of new drugs by physicians: a survival analysis. BMC Health Serv Res 2012, 12:56.

31. Chauhan D, Mason A: Factors affecting the uptake of new medicines in secondary care - a literature review. J Clin Pharm Ther 2008, 33(4):339-48.

32. Ohlsson $\mathrm{H}$, Merlo J: Is physician adherence to prescription guidelines a general trait of health care practices or dependent on drug type? - A multilevel logistic regression analysis in South Sweden. Pharmacoepidemiol Drug Saf 2009, 18(8):682-90.

33. Di Carlo A, Lamassa M, Baldereschi M, Pracucci G, Basile AM, Wolfe CD, Giroud M, Rudd A, Ghetti A, Inzitari D, European BIOMED Study of Stroke Care Group: Sex differences in the clinical presentation, resource use, and 3-month outcome of acute stroke in Europe: data from a multicenter multinational hospital-based registry. Stroke 2003, 34(5):1114-9.

34. Hjalmarsson C, Bokemark L, Manhem K, Mehlig K, Andersson B: The effect of statins on acute and long-term outcome after ischemic stroke in the elderly. Am J Geriatr Pharmacother 2012, 10(5):313-22.

35. Mauskop A, Borden WB: Predictors of statin adherence. Curr Cardiol Rep 2011, 13(6):553-8.

36. Lummis HL, Sketris IS, Gubitz GJ, Joffres MR, Flowerdew GJ: Medication persistence rates and factors associated with persistence in patients following stroke: a cohort study. BMC Neurol 2008, 8:25.

37. Savarese G, Gotto AM Jr, Paolillo S, D'Amore C, Losco T, Musella F, Scala O, Marciano C, Ruggiero D, Marsico F, De Luca G, Trimarco B, Perrone-Filardi P: Benefits of statins in elderly subjects without established cardiovascular disease. a meta-analysis. J Am Coll Cardiol 2013, 14 [E-pub ahead of print]

38. Nì Chròinìn D, Nì Chròinìn C, Callaly EL, Hannon N, Kelly L, Marnane M, Merwick A, Shhehan O, Hirgan G, Duggan J, Kyne L, Dolan E, Moore A Williams D, Kelly PJ: Suboptimal lipid management in ischaemic stroke and TIA patients - The North Dublin population stroke study. CerebrovasC Dis 2013, 35(Suppl 3)

39. Kablau M, Kreisel SH, Sauer T, Binder J, Szabo K, Hennerici MG, Kern R: Predictors and early outcome of hemorrhagic transformation after acute ischemic stroke. Cerebrovasc Dis 2011, 32(4):334-41.

40. Goldstein LB, Amarenco P, Szarek M, Callahan A 3rd, Hennerici M, Sillesen H, Zivin JA, Welch KM, SPARCL Investigators: Hemorrhagic stroke in the Stroke Prevention by Aggressive Reduction in Cholesterol Levels study. Neurology 2008, 70(24 Pt 2):2364-70.

41. LaRosa JC, Pedersen TR, Somaratne R, Wasserman SM: Safety and effect of very low levels of low-density lipoprotein cholesterol on cardiovascular events. Am J Cardiol 2013, 111(8):1221-9.

42. Vauthey C, de Freitas GR, van Melle G, Devuyst G, Bogousslavsky J: Bette outcome after stroke with higher serum cholesterol levels. Neurology 2000, 54(10):1944-9.

43. Iso H, Jacobs DR Jr, Wentworth D, Neaton JD, Cohen JD: Serum cholesterol levels and six-year mortality from stroke in 350,977 men screened for the Multiple Risk Factor Intervention Trial. N Eng J Med 1989, 320(14):904-10.

44. Suzuki K, Izumi M, Sakamoto T, Hayashi M: Blood pressure and total cholesterol level are critical risks especially for hemorrhagic stroke in Akita, Japan. Cerebrovasc Dis 2011, 31(1):100-6.
45. Sillesen H, Amarenco P, Hennerici MG, Callahan A, Goldstein LB, Zivin J, Messig M, Welch KM, Stroke Prevention by Aggressive Reduction in Cholesterol Levels Investigators: Atorvastatin reduces the risk of cardiovascular events in patients with carotid atherosclerosis: a secondary analysis of the Stroke Prevention by Aggressive Reduction in Cholesterol Levels (SPARCL) trial. Stroke 2008, 39(12):3297-302.

46. Day JS, Policeni BA, Smoker WR, Dobre MC, Zhang Y, Leira EC, Davis PH, Chen S, Olalde H, Adams HP Jr: Previous statin use is not associated with an increased prevalence or degree of gradient-echo lesions in patients with acute ischemic stroke or transient ischemic attack. Stroke 2011, 42(2):354-8.

47. Haussen DC, Henninger N, Kumar S, Selim M: Statin use and microbleeds in patients with spontaneous intracerebral hemorrhage. Stroke 2012, 43(10):2677-81.

48. Furie K: High-dose statins should only be used in atherosclerotic strokes. Stroke 2012, 43(7):1994-5

49. Alvarez-Sabìn J, Huertas R, Quintana M, Delgado P, Ribo M, Molina CA, Montaner J: Prior statin use may be associated with improved stroke outcome after tissue plasminogen activator. Stroke 2007, 38(3):1076-8.

50. Uyttenboogaart M, Koch MW, Koopman K, Vroomen PC, Luijckx GJ, De Keyser J: Lipid profile, statin use, and outcome after intravenous thrombolysis for acute ischemic stroke. J Neurol 2008, 255(6):875-80.

51. Meier N, Nedeltchev K, Brekenfeld C, Galimanis A, Fischer U, Findling O, Remonda L, Schroth G, Mattle HP, Arnold M: Prior statin use, intracranial hemorrhage and outcome after intra-arterial thrombolysis for acute ischemic stroke. Stroke 2009, 40(5):1729-37.

52. Engelter ST, Soinne L, Ringleb P, Sarikaya H, Bordet R, Berrouschot J, Odier C, Arnold M, Ford GA, Pezzini A, Zini A, Rantanen K, Rocco A, Bonati LH, Kellert L, Strbian D, Stoll A, Meier N, Michel P, Baumgartner RW, Leys D, Tatlisumak T, Lyrer PA: IV thrombolysis and statins. Neurology 2011, 77(9):888-95.

53. Cappellari M, Deluca C, Tinazzi M, Tomerelli G, Carletti M, Fiaschi A, Bovi P, Moretto G: Does statin in the acute phase of ischemic stroke improve outcome after intravenous thrombolysis? A retrospective study. J Neurol Sci 2011, 308(1-2):128-34.

54. Cappellari M, Bovi P, Moretto G, Zini A, Nencini P, Sessa M, Furlan M, Pezzini A, Orlandi G, Paciaroni M, Tassinari T, Procaccianti G, Di Lazzaro V, Bettoni L, Gandolfo C, Silvestrelli G, Rasura M, Martini G, Melis M, Calloni MV, Chiodo-Grandi F, Beretta S, Guarino M, Altavista MC, Marcheselli S, Galletti G, Adobbati L, Del Sette M, Mancini A, Orrico D, et al: The THRombolysis and STatins (THRaST) study. Neurology 2013, 80(7):655-61

55. Ní Chròinín D, Callaly E, Duggan J, Merwick A, Hannon N, MB, Sheehan O, Marnane M, Horgan G, Williams EB, Harris D, Kyne L, McCormack PM, Moroney J, Grant T, Williams D, Daly L, Kelly PJ: Association Between Acute Statin Therapy, Survival, and Improved Functional Outcome After Ischemic Stroke The North Dublin Population Stroke Study. Stroke 2011, 42(4):1021-9.

56. Lakhan SE, Bagchi S, Hofer M: Statins and clinical outcome of acute ischemic stroke: a systematic review. Int Arch Med 2010, 3:22.

57. Hackam D, Woodward M, Newby K, Bhatt D, Shao M, Smith EE, Donner A, Mamdani M, Douketis JD, Arima H, Chalmers J, MacMahon S, Tirschwell DL, Psaty BM, Bushnell CD, Aguilar MI, Capampangan DJ, Werring DJ, De Rango P, Viswanathan A, Danchin N, Cheng CL, Yang YH, Verdel BM, Lai MS, Kennedy J, Uchiyama S, Yamaguchi T, Ikeda Y, Mrkobrada M: Statins and Intracerebral Hemorrhage Collaborative Systematic Review and Meta-Analysis. Circulation 2011, 124(20):2233-42.

58. Montaner J, Chacòn, Krupinski J, Rubio F, Millàn M, Molina CA, Hereu P, Quintana M, Alvarez-Sabín J: Simvastatin in acute phase of ischemic stroke: a safety and efficacy pilot trial. Eur J Neurol 2008, 15(1):82-90.

59. D’Amelio M, Terruso V, Famoso G, Ragonese P, Aridon P, Savettieri G Cholesterol levels and risk of hemorrhagic transformation after acute ischemic stroke. Cerebrovasc Dis 2011, 32(3):234-8.

60. Selim MH, Molina CA: High-dose statin for every stroke. The good, the bad, and the unknown. Stroke 2012 Jul, 43(7):1996-7.

61. Adams RJ, Albers G, Alberts MJ, Benavente O, Furie K, Goldstein LB, Gorelick P, Halperin J, Harbaugh R, Johnston SC, Katzan I, Kelly-Hayes M, Kenton EJ, Marks M, Sacco RL, Schwamm LH, American Heart Association, American Stroke Association: Update to the AHA/ASA recommendations for the prevention of stroke in patients with stroke and transient ischemic attack. Stroke 2008, 39(5):1647-52 
62. Adams HP, del Zoppo G, Alberts MJ, Bhatt DL, Brass L, Furlan A, Grubb RL, Higashida RT, Jauch EC, Kidwell C, Lyden PD, Morgenstern LB, Qureshi Al, Rosenwasser RH, Scott PA, Wijdicks EF, American Heart Association/ American Stroke Association Stroke Council, American Heart Association/ American Stroke Association Clinical Cardiology Council, American Heart Association/American Stroke Association Cardiovascular Radiology and Intervention Council, Atherosclerotic Peripheral Vascular Disease Working Group, Quality of Care Outcomes in Research Interdisciplinary Working Group: Guidelines for the early management of adults with ischemic stroke. Circulation 2007, 115(20):e478-534.

doi:10.1186/1471-2377-14-53

Cite this article as: Canavero et al:: Clinical factors associated with

statins prescription in acute ischemic stroke patients: findings from the Lombardia Stroke Registry. BMC Neurology 2014 14:53.

\section{Submit your next manuscript to BioMed Central and take full advantage of:}

- Convenient online submission

- Thorough peer review

- No space constraints or color figure charges

- Immediate publication on acceptance

- Inclusion in PubMed, CAS, Scopus and Google Scholar

- Research which is freely available for redistribution 